\title{
A Paradox of Eventual Linearizability in Shared Memory
}

\author{
Rachid Guerraoui \\ EPFL, Switzerland
}

\author{
Eric Ruppert \\ York University, Canada
}

\begin{abstract}
This paper compares, for the first time, the computational power of linearizable objects with that of eventually linearizable ones. We present the following paradox. We show that, unsurprisingly, no set of eventually linearizable objects can (1) implement any non-trivial linearizable object, nor (2) boost the consensus power of simple objects like linearizable registers. We also show, perhaps surprisingly, that any implementation of an eventually linearizable complex object like a fetch\&increment counter (from linearizable base objects), can itself be viewed as a fully linearizable implementation of the same fetch\&increment counter (using the exact same set of base objects).
\end{abstract}

\section{Categories and Subject Descriptors}

D.1.3 [Programming Techniques]: Concurrent Programming

\section{Keywords}

Concurrent; asynchronous; linearizable; consistency; waitfree; obstruction-free; fetch-and-increment; consensus

\section{INTRODUCTION}

A central activity in shared-memory computing is that of raising the abstraction level of synchronization primitives by building, in software, higher-level inter-process communication objects than those provided in hardware. The goal is usually to ensure that, even if the constructed objects have richer semantics, they appear as if they were provided in hardware. In particular, every process should be able to access the shared object independently of contention, i.e., even when other processes may be accessing the same object. This property, called wait-freedom [9], says that if we build, for instance, a fetch\&increment counter in software, then every process should be able to increment the counter and get its new value, independently of whether other processes that are accessing the

Permission to make digital or hard copies of all or part of this work for personal or classroom use is granted without fee provided that copies are not made or distributed for profit or commercial advantage and that copies bear this notice and the full citation on the first page. Copyrights for components of this work owned by others than ACM must be honored. Abstracting with credit is permitted. To copy otherwise, or republish, to post on servers or to redistribute to lists, requires prior specific permission and/or a fee. Request permissions from permissions@ acm.org. PODC'14, July 15-18, 2014, Paris, France.

Copyright 2014 ACM 978-1-4503-2944-6/14/07 ...\$15.00.

http://dx.doi.org/10.1145/2611462.2611484 counter concurrently have been swapped or paged out. This requirement rules out the usage of locks. However, in order to provide the illusion of a single shared object, processes must synchronize using underlying hardware primitives, such as compare\&swap. This illusion, in turn, is typically captured by the property of being linearizable [11]. This property says that even if they are actually performed concurrently, the operations issued on that object appear as if they are executed sequentially.

Ensuring either linearizability or wait-freedom alone is simple. What is challenging, and sometimes considered too expensive, is to ensure both at the same time. Two major research directions have been considered to reduce the difficulty. The first is to weaken wait-freedom and devise algorithms that "simply" ensure lock-freedom, obstruction-freedom, or even use locks in specific portions of the code [10]. The rationale here is that the conditions under which the operating system swaps out a process for a long period of time do not happen very often. A lot of theoretical work has been devoted in the last decades to this avenue of research. The second approach is to weaken linearizability: for some applications, weaker consistency conditions suffice [19]. Consider a shared fetch\&increment abstraction used to count references of objects in a concurrent setting. As pointed out, this would typically be implemented in software using the system's compare\&swap objects. If several compare\&swap tentatives fail due to unusually high contention, it may be acceptable to return a temporary value of the counter, as long as, eventually, all increments of concurrent processes are taken into account in some future value of the counter. In other words, in the implementation, if a process is taking too long to synchronize perfectly, because of contention, it could give up trying to get the most up-to-date value and (a) do its increment locally, making sure later that its increments are eventually counted, and (b) get a value of the counter to return that is lower than the true value, assuming that the process will get information about concurrent increments later.

The notion of eventual consistency [19] aims to capture precisely this notion of "intermittent inconsistency". The approach is analogous to the way self-stabilization was defined, namely stating that the system should eventually work correctly forever beyond some stabilization time, with the goal of modelling intermittent failures and the return of the system to normal after the failure. Obviously, if instead of consistency, implementations only need to implement an eventual form of it, they would offer weaker guarantees. In our example above, the value returned by the counter might be temporarily inconsis- 
tent with the eventual value. But would these implementations be easier to implement? Maybe surprisingly, we show that the answer is no in some situations. More specifically, we highlight the following paradox.

- On the one hand, unsurprisingly, eventual linearizability is strictly weaker than linearizability in the following two senses. (1) No set of eventually linearizable objects can implement any non-trivial linearizable object. (A trivial object is one that can be implemented without inter-process communication.) This is true even if we only require the resulting linearizable object to be obstruction-free, whereas the eventually linearizable base objects are waitfree. (2) No set of eventually linearizable objects can boost the consensus power of simple linearizable objects like registers, i.e., help solve consensus among two processes in conjunction with linearizable registers. In short, eventually linearizable objects can neither implement linearizable ones (1) nor augment the power of shared linearizable registers (2).

- On the other hand, eventual linearizability can be as hard to implement as linearizability: any implementation of an eventually linearizable fetch\&increment counter (from a set of linearizable base objects), yields an implementation of a fully linearizable fetch\&increment counter (from the same set of base objects). Roughly speaking, this is because a fetch\&increment object continues to require synchronization forever. In contrast, such a result does not hold for objects that require synchronization only in the beginning of an execution: for example, consensus and test\&set objects are trivial to implement in an eventually linearizable manner. Thus, an eventually linearizable implementation of consensus (respectively, test\&set) will clearly not yield a linearizable implementation of consensus (respectively, test\&set).

In fact, we show that any algorithm $A$ that implements an eventually linearizable fetch\&increment object, starting from a given initial state of the object, can itself be viewed as an algorithm $A^{\prime}$ that implements a fully linearizable fetch\&increment object, simply starting from a different initial state of the counter than $A$. Basically, the variables of $A^{\prime}$ are those of $A$, initialized after a global "stabilization time" of eventual linearizability. Interestingly, this is in spite of the fact that the stabilization time is not a priori fixed: it could be different in different executions (and this is allowed by our definition of eventual linearizability). The main difficulty of the proof is to show that there must exist a stable configuration $C$ of algorithm $A$ where every possible run that passes through $C$ has already stabilized at $C$. (Then, we initialize the variables of $A$ as they are in $C$ to get $A^{\prime}$.)

\section{RELATED WORK}

Eventual consistency originated in the context of replicated systems where updates to the replicas are propagated using gossipping to improve latency and tolerate asynchrony and partitions $[4,6,18]$. Because gossipping is used, replicas are not always consistent, but eventually become so if the updates stop. More recently, researchers argued eventual consistency is a reasonable alternative to consistency in the cloud context. The argument is that consistency does not scale and is not necessary for many applications, e.g., $[1,17,19]$. So far, research on even- tual consistency has focused on describing systems that implement some form of eventual consistency.

Some attempts have been made to formalize the semantics of eventual consistency. In $[14,15]$, for instance, the focus was on abstract properties, while in [2] the goal was to show how to verify replication schemes that ensure only eventual consistency. However, little effort has been devoted to theoretical studies that would precisely measure the power and limitations of eventual consistency (beyond artifacts related to specific implementations). Two notable exceptions are [16] and [5].

In [16], Serafini et al. introduced the concept of eventual linearizability and addressed the problem of the weakest failure detector to implement objects for which some operations are linearizable and some operations are not. In a sense, their work is orthogonal to ours. The main common feature is the definition of eventual linearizability. There are however two differences between our definition of an eventually linearizable implementation and the definition used by Serafini et al. The first is a minor difference. Serafini et al. used a timed model of computation, and talked about stabilizing after time $t$ rather than after $t$ events. This is due to their underlying message-passing model equipped with failure detectors where timing assumptions are used, while we consider a totally asynchronous sharedmemory model, where time plays no role. The second, more important difference, has to do with quantifiers. Serafini et al. defined eventual linearizability of an implementation by saying that there is a single time $t$ such that all executions stabilize by time $t$. We do not require a single $t$ for all executions: the number of events before executions stabilize may vary, and even be unbounded. Serafini et al. motivate their choice of focusing only on finite executions by arguing that any finite history is trivially linearizable after some $t$ (because $t$ can be chosen large enough to satisfy the definition vacuously), and thus the notion of eventual linearizability makes sense only if there is a single $t$ for all executions. We take a different approach. We consider infinite executions, which do not trivially satisfy the property of being $t$-linearizable for some $t$. We made this choice following an analogy with the literature on self-stabilization, which also deals with a property describing eventual good behaviour.

Dubois et al. [5] consider a message-passing environment and compare the weakest failure detector to implement total order broadcast with the weakest failure detector to implement eventual total order broadcast. Their results do not provide a comparison between linearizable and eventually linearizable implementations of specific objects. We show in this paper that some eventually linearizable objects are trivial to implement in an asynchronous system (and would thus not require any failure detector), whereas others are as hard to implement as their linearizable counterparts. Note that the fact that two abstractions have the same weakest failure detectors does not mean that any implementation of the first is also an implementation of the second (which is what we show for fetch\&increment objects in this paper). For instance, [3] shows that two object types $T_{1}$ and $T_{2}$ can have the same weakest failure detectors even if the consensus number of $T_{1}$ is greater than that of $T_{2}$, meaning that $T_{2}$ cannot implement $T_{1}$.

To our knowledge, this paper is the first to compare the computational power of linearizable and eventually linearizable objects in a shared-memory context. 


\section{MODEL, DEFINITIONS AND PROPERTIES}

A shared-memory system consists of a collection of processes that communicate by accessing shared objects of various types. Each type of object has a sequential specification, which consists of

- a set $Q$ of possible states,

- a set of possible initial states $Q_{0} \subseteq Q$,

- a set $I N V$ of possible operation invocations,

- a set $R E S$ of possible operation response values, and

- a transition relation $\delta \subseteq Q \times O P \times R E S \times Q$.

Intuitively, if $\left(q, o p, r, q^{\prime}\right) \in \delta$, it means that when the object is in state $q$ and operation $o p$ is applied to it, the object can return the response $r$ and move into state $q^{\prime}$. We consider the name of an operation in $O P$ to include all of the operation's arguments. A type $T$ is deterministic if, for each state $q$ and each operation $o p$, there is a unique result $r$ and state $q^{\prime}$ such that $\left(q, o p, r, q^{\prime}\right) \in \delta$. A type $T$ has finite non-determinism if, for each state $q$ and each operation $o p$, there are finitely many pairs $\left(r, q^{\prime}\right)$ such that $\left(q, o p, r, q^{\prime}\right) \in \delta$.

An event is a tuple $\langle p, o, x\rangle$ where $p$ is a process name, $o$ is the name of an object of some type $T$ and $x$ is either an invocation or response for type $T$. A computation of the distributed system is described by a sequence of such events, called a history. We use $H \mid o$ to denote the subsequence of history $H$ consisting of events at object $o$ and $H \mid p$ to denote the subsequence $H$ consisting of events performed by process $p$. A history is sequential if it consists of operation invocation and response events, starting with an invocation, where each invocation $\langle p, o, i n v\rangle$ (except possibly the last, if the history is finite) is immediately followed by a matching response $\langle p, o, r e s\rangle$. Any history $H$ mentioned in this paper is assumed to be well-formed, i.e., the subsequence $H \mid p$ is sequential for each process $p$. An operation consists of an invocation event and its matching response event (if it exists).

A sequential history $H$ is legal if, for each object $o$, the subsequence $H \mid o=\left\langle p_{1}, o, i_{1}\right\rangle,\left\langle p_{1}, o, r_{1}\right\rangle,\left\langle p_{2}, o, i_{2}\right\rangle$, $\left\langle p_{2}, o, r_{2}\right\rangle, \ldots$ satisfies the following with respect to $o$ 's sequential specification $\left(Q, Q_{0}, I N V, R E S, \delta\right)$ : there is a sequence of states $q_{0}, q_{1}, \ldots$ in $Q$ such that $q_{0} \in Q_{0}$ and, for each $j \geq 0,\left(q_{j}, i_{j}, r_{j}, q_{j+1}\right) \in \delta$.

An implementation of an object of type $T=\left(Q, Q_{0}\right.$, $I N V, R E S, \delta)$ provides, for each $q_{0} \in Q_{0}$, a programme that each process can follow to perform each operation $o p$ in $I N V$. When the programme terminates, it outputs a result in $R E S$. Since we focus on a shared-memory model, the programme can make use of shared base objects: it can invoke an operation on a base object and await a response from it. In a concurrent execution of the implementation, each process repeatedly executes the programme to perform an operation until the programme generates a response to the operation, and the steps of different processes may be interleaved in an arbitrary way. Each execution defines a history, the sequence of invocations and responses on the object of type $T$. In Section 3.1 , we define eventual linearizability, which is a correctness condition for an implementation, that constrains the set of possible histories that can arise from executions of the implementation.

An implementation is wait-free if each operation completes within a finite number of steps of the process performing it. An implementation is non-blocking if, when- ever some operation is pending and processes continue to take steps, some operation is eventually completed. (Some authors call the non-blocking progress property lock-freedom.) An implementation is obstruction-free if, there is no execution where only one process takes steps in an infinite suffix without completing its operation.

Generally, the focus of distributed computing research is on the interprocess communication, and the computational power of the processes is not specified. For this work, we assume that the programmes are written in a programming language that has equivalent power to the Turing machine model (but includes a mechanism for accessing the shared base objects). Correspondingly, we assume that the transition relations of object type specifications are Turing-computable.

\subsection{Eventual Linearizability}

We now give several definitions that are used in defining eventual linearizability. These are based on the definitions in [16], but differ in the ways described in Section 2. The first property, weak consistency, ensures that operations cannot return responses that are "out of left field," even during the initial period when absolute consistency is not enforced. In particular, the response to each operation should take into account earlier operations done by the same process and should be a possible response if the process knows only about a subset of the operations performed by other processes.

Definition 1. A history $H$ is weakly consistent if, for each operation op that has a response in $H$, there is a legal sequential history $S$ that

- contains only operations that are invoked in $H$ before op terminates,

- contains all operations that are performed by the same process that does op and precede op in $H$, and

- ends with the same response to op as in $H$.

Now we define the notion of being linearizable "after the first $t$ events have occurred."

DeFinition 2. Let $H$ be a history and $t$ be a natural number. Let $H^{\prime}$ be the suffix of $H$ obtained by removing the first $t$ events. Then, a legal sequential history $S$ is called a $t$-linearization of $H$ if

- each operation invoked in $S$ is invoked in $H$,

- each operation completed in $H$ is completed in $S$,

- if op ${ }_{1}$ 's response is before op ${ }_{2}$ 's invocation and both of these events are in $H^{\prime}$ and $o p_{2}$ is in $S$, then $o_{1}$ precedes $\mathrm{op}_{2}$ in $S$, and

- each operation that has a response in $H^{\prime}$ has the same response in $S$.

$A$ history is t-linearizable if it has a t-linearization.

Definition 3. A history is eventually linearizable if it is weakly consistent and t-linearizable for some $t$.

Definition 4. An implementation of an object is eventually linearizable if every history is eventually linearizable. (Note that different histories can be t-linearizable for different values of $t$.)

\subsection{Basic Properties}

We start with some easy properties of $t$-linearizability. 
LEMmA 5. If a history $H$ is t-linearizable, then it is also $t^{\prime}$-linearizable for any $t^{\prime}>t$.

Proof. If $S$ is a $t$-linearization of $H$, then $S$ is also a $t^{\prime}$-linearization of $H$.

Lemma 6. If a history $H$ is t-linearizable, then every prefix of $H$ is also t-linearizable.

Sketch of proof. Let $S$ be a $t$-linearization of $H$. A prefix of $H$ of length at most $t$ is trivially $t$-linearizable. So, consider a prefix $H_{1} H_{2}$ of $H$ where $\left|H_{1}\right|=t$. Let $S_{1}$ be the shortest prefix of $S$ containing all operations that terminate in $H_{2}$. Let $S_{2}$ be an arbitrary permutation of the operations that terminate in $H_{1}$ but do not appear in $S_{1}$ (with the responses they would have if executed in that order after $S_{1}$ ). Then, it is easy to check that $S_{1} S_{2}$ is a $t$-linearization of $H_{1} H_{2}$

Serafini et al. [16] prove the following proposition using their slightly different definition of $t$-linearizability. Note, however, that the proof holds only for histories involving a finite number of objects (an assumption that should have been included in [16]).

Lemma 7. (Proved in [16]) A history $H$ involving a finite number of objects is t-linearizable for some $t$ if and only if, for each object o, there is a $t_{o}$ such that $H \mid o$ is $t_{o}$-linearizable.

The proof of Lemma 7 is based on the proof in [11] that linearizability is a local property, and it carries over to our definition. For the "only if" direction, we can take $t_{o}=t$. For the "if" direction, we choose $t$ large enough so that the first $t$ events of $H$ include the first $t_{o}$ events of $H \mid o$ for each $o$. This is possible because the set of objects $O$ is finite.

The proof of the following lemma in [16] has a small error, so for completeness, we provide a detailed proof.

LEMma 8. A history $H$ is weakly consistent if and only if, for each object $o, H \mid o$ is weakly consistent.

Proof. Suppose $H$ is weakly consistent. Let $o$ be any object and $o p$ be any operation with a response in $H \mid o$. Since $H$ is weakly consistent, there is a sequential history $S$ that satisfies Definition 1 for op in $H$. Then, $S \mid o$ satisfies Definition 1 for $o p$ in $H \mid o$. So, $H \mid o$ is weakly consistent.

Now, suppose $H \mid o$ is weakly consistent for each object $o$. Let $o p$ be any operation that has a response in $H$. Let $p$ be the process that performs op and $o$ be the object $o p$ is performed on. Let $S$ be the legal sequential history that satisfies Definition 1 for op in $H \mid o$. Let $S^{\prime}$ be obtained from $S$ by inserting at the beginning of $S$ any operations performed by $p$ before $o p$ in $H$ on objects other than $o$, with responses as dictated by the objects' sequential specifications. Then, $S^{\prime}$ satisfies Definition 1 for op in $H$. Thus, $H$ is weakly consistent.

The next proposition is immediate from Lemma 7 and 8 .

Proposition 9. A history $H$ that involves a finite number of objects is eventually linearizable if and only if, for each object o, $H \mid o$ is eventually linearizable.

Proposition 9 does not hold for some histories that use infinitely many objects. For example, consider the following sequential history $H$ that uses read/write registers
$R_{1}, R_{2}, \ldots$, all initialized to the value 0 .

$\left\langle p, R_{1}\right.$, write $\left.(1)\right\rangle,\left\langle p, R_{1}\right.$, ack $\rangle,\left\langle q, R_{1}\right.$, read $\rangle,\left\langle q, R_{1}, 0\right\rangle$,

$\left\langle p, R_{2}\right.$, write $\left.(1)\right\rangle,\left\langle p, R_{2}\right.$, ack $\rangle,\left\langle q, R_{2}\right.$, read $\rangle,\left\langle q, R_{2}, 0\right\rangle$, $\left\langle p, R_{3}\right.$, write $\left.(1)\right\rangle,\left\langle p, R_{3}\right.$, ack $\rangle,\left\langle q, R_{3}\right.$, read $\rangle,\left\langle q, R_{3}, 0\right\rangle, \ldots$

If $t_{i}$ is chosen so that the response to the read of $R_{i}$ occurs before the $t_{i}$ th event of $H$, then $H \mid R_{i}$ is $t_{i}$-linearizable and it is easy to see that $H \mid R_{i}$ is weakly consistent, so $H \mid R_{i}$ is eventually linearizable for every $i$. However, $H$ is not eventually linearizable: for any $t$, there is an $i$ such that the write to $R_{i}$ is invoked after the $t$ th event of $H$, and $H \mid R_{i}$ is not $t$-linearizable, so $H$ is not $t$-linearizable.

A safety property is a set of histories that is non-empty, prefix-closed and limit-closed. A liveness property is a set of histories such that every finite history is the prefix of some history in the set. The following lemma follows easily from the definition of weak consistency.

\section{LEMMA 10. Weak consistency is a safety property.}

Proof. The empty execution (consisting of no events) vacuously satisfies Definition 1, so the set of weakly consistent histories is non-empty.

Next, we prove that the set of weakly consistent histories is prefix-closed. Suppose $H$ is weakly consistent. Let $H^{\prime}$ be a prefix of $H$. Let $o p$ be an operation that terminates in $H^{\prime}$. Let $S$ be the sequential history that satisfies definition 1 for $o p$ in $H$. Then $S$ also satisfies Definition 1 for $o p$ in $H^{\prime}$. Thus, $H^{\prime}$ is weakly consistent.

To show the set of weakly consistent histories is limitclosed, let $H_{1}, H_{2}, \ldots$ be an infinite sequence of histories such that, for each $i, H_{i}$ is weakly consistent and $H_{i}$ is a prefix of $H_{i+1}$. We show that $H=\lim _{i \rightarrow \infty} H_{i}$ is weakly consistent. Let $o p$ be an operation that terminates in $H$. Then there exists an $i$ such that op terminates in $H_{i}$. Let $S$ be a sequential history that satisfies Definition 1 for $o p$ in $H_{i}$. Then, $S$ also satisfies Definition 1 for $o p$ in $H$.

0 -linearizability is equivalent to linearizability [11]. For deterministic objects, Lynch [13] proved that linearizability is a safety property. The proof extends easily to objects with finite non-determinism [8]. However, linearizability is not a safety property for objects with infinite non-determinism [8].

For $t>0, t$-linearizability is not a safety property, even for deterministic types. Consider a fetch\&increment object, which stores a natural number and provides a single operation, fetch\&inc, which adds one to the value stored and returns the old value. Consider the infinite sequential history which uses a single fetch\&increment object $X$ initialized to the value 0 .

$\langle p, X$, fetch\&inc $\rangle,\langle p, X, 0\rangle,\langle q, X$, fetch\&inc $\rangle,\langle q, X, 0\rangle$, $\langle q, X$, fetch\&inc $\rangle,\langle q, X, 1\rangle,\langle q, X$, fetch\&inc $\rangle,\langle q, X, 2\rangle, \ldots$ Every finite prefix is $t$-linearizable if event $t$ is the response to the first operation: the $t$-linearization moves the first operation to the end. However, the whole infinite history is not $t$-linearizable.

Thus, $t$-linearizability is neither a safety nor a liveness property. However, the property of being $t$-linearizable for some $t$ is trivially a liveness property: for any finite history $H$, just take $t$ to be equal to the number of events in $H$, and then any permutation of the operations in $H$ yields a legal, sequential history that is a $t$-linearization of $H$. Thus, eventual linearizability is the intersection of a safety property (weak consistency) and a liveness property (being $t$-linearizable for some $t$ ). 


\subsection{Using Registers to Obtain Weak Consistency}

Our definition of eventual linearizability combines a safety property (weak consistency) and a liveness property (being $t$-linearizable for some $t$ ). If our underlying system includes linearizable read/write registers, then we can easily modify any non-blocking implementation that satisfies the liveness property to also satisfy the safety property.

Proposition 11. Consider a system that includes linearizable registers as base objects. Let $T$ be an object type with finite nondeterminism. There is an eventually linearizable non-blocking implementation of $T$ if and only if there is a non-blocking implementation of $T$ such that for every history there is a $t$ such that the history is $t$ linearizable.

This proposition is proved in detail in the appendix. The basic idea is to announce all operations by writing them to shared memory. Then, when a response to an operation has been computed, a process reads all announced operations and returns the response only if it can verify that the response does not violate weak consistency. If a violation of weak consistency is detected, the process is free to return any response that is consistent with just its own prior operations.

\section{EVENTUAL LINEARIZABILITY IS WEAK}

It is fairly easy to prove that eventually linearizable objects by themselves are useless for implementing linearizable objects. The intuition is that a linearizable implementation must eventually produce the correct output for some operations, but if the eventually linearizable base objects used by the implementation are still behaving badly when that output is produced, that output can be incorrect. The following theorem says that there is no linearizable implementation of any non-trivial object that is useful for inter-process communication from any collection of eventually linearizable objects.

THEOREM 12. For $n \geq 2$, the following are equivalent for any type $T$ with finite non-determinism.

1. There is an n-process linearizable wait-free implementation of $T$ using no shared objects.

2. There is an $n$-process linearizable obstruction-free implementation of $T$ using no shared objects.

3. There is an n-process linearizable wait-free implementation of $T$ from some collection of eventually linearizable objects.

4. There is an n-process linearizable obstruction-free implementation of $T$ from some collection of eventually linearizable objects.

Proof. It is trivial to see that $1 \Rightarrow 3 \Rightarrow 4$ and $1 \Rightarrow 2$ $\Rightarrow 4$. So, it remains to prove that $4 \Rightarrow 1$.

Suppose there is an obstruction-free linearizable implementation $I$ of an object of type $T$ from some collection of eventually linearizable objects for $n$ processes $p_{1}, \ldots p_{n}$. We construct an $n$-process wait-free linearizable implementation $I^{\prime}$ of an object of type $T$ simply by replacing each shared object $o$ by $n$ local copies $o_{1}, \ldots, o_{n}$.
(Since we assume that transition functions are Turingcomputable, a process can simulate the object in its local memory.) Whenever process $p_{i}$ must perform an operation $o p$ on shared object $o$ according to $I, p_{i}$ instead performs op on its local copy $o_{i}$.

Then, any finite history $H$ of $I^{\prime}$ is also a possible finite history of $I$ since the eventually linearizable objects used by $I$ can return arbitrary answers (that satisfy weak consistency) in any finite prefix of an execution. (Note that using a local copy of each object ensures the responses satisfy weak consistency.) Thus, every finite history $H$ of $I^{\prime}$ must be linearizable, since $I$ guarantees linearizability. Since linearizability is a safety property for $T$ (which is assumed to have finite non-determinism), this means that every history of $I^{\prime}$ is linearizable.

It remains to show that $I^{\prime}$ is wait-free. Consider any execution $\alpha$ of $I^{\prime}$ and any process $p_{i}$ that takes infinitely many steps in $\alpha$. Since there is no communication between processes in $\alpha, p_{i}$ cannot distinguish $\alpha$ from $\alpha \mid p_{i}$, where $p_{i}$ runs solo. But $\alpha \mid p_{i}$ is also a solo execution of $I$ and $I$ is obstruction-free, so every operation that $p_{i}$ invokes in this execution must terminate.

Theorem 12 allows us to characterize the trivial deterministic types that have linearizable implementations from eventually linearizable objects as follows.

Definition 13. A deterministic type $T$ is called trivial if and only if there is a computable function $r$ that maps each initial state $q_{0}$ and operation op to a response $r\left(q_{0}, o p\right)$ that is the correct response to op for every state reachable from $q_{0}$.

Proposition 14. A deterministic type T has a linearizable obstruction-free implementation for 2 processes from some collection of eventually linearizable objects if and only if $T$ is trivial.

Proof. $(\Leftarrow)$ : Suppose $T$ is trivial. For any initial state $q_{0}$, an object of type $T$ initialized to state $q_{0}$ can be implemented by having op simply return $r\left(q_{0}, o p\right)$.

$(\Rightarrow)$ : Suppose there is an obstruction-free linearizable implementation from eventually linearizable objects. By Theorem 12, there is also an obstruction-free linearizable implementation using no shared objects at all for two processes $p_{1}$ and $p_{2}$. To compute $r\left(q_{0}, o p\right)$, we simply run the programme for $p_{1}$ that implements $o p$ for an object initialized to state $q_{0}$ until it produces a response. Let $\alpha$ be this solo execution of the implementation. Let $q$ be any state that can be reached from $q_{0}$ via some sequence of operations. We must show that $r\left(q_{0}, o p\right)$ is the correct response for $o p$ when an object of type $T$ is in state $q$. Consider an execution $\beta$ of the implementation in which process $p_{2}$ runs solo, performing a sequence of operations that takes the implemented object from the initial state $q_{0}$ to state $q$. (This uses the assumption that $T$ is deterministic, so that we can force the implemented object into any reachable state.) Since the implementation uses no shared objects, $\beta \alpha$ is a also possible execution of the implementation. Since this execution is linearizable, and $p_{1}$ returns $r\left(q_{0}, o p\right)$ as the response for $o p$ in $\alpha$, there must be a transition $\left(q, o p, r\left(q_{0}, o p\right), q^{\prime}\right)$ in the transition relation that specifies type $T$.

In particular, Proposition 14 says that even weak objects like read/write registers do not have linearizable implementations from any collection of eventually linearizable objects. 


\section{Eventual Linearizability is Still Weak Even With Linearizable Registers}

Theorem 12 tells us that if $T$ is a non-trivial type (i.e., cannot be implemented linearizably without any communication) then a linearizable implementation of $T$ cannot be built from eventually linearizable objects. But what if we also have linearizable registers available? Then, could we build a linearizable object of type $T$ ? We use a valency argument [7] to show the answer is still no if $T$ is strong enough to solve two-process consensus.

Proposition 15. Let $T$ be any object type that can solve wait-free two-process consensus. There is no waitfree linearizable implementation of $T$ from linearizable registers and any collection of eventually linearizable objects (of any types).

Proof. Suppose there were such an implementation to derive a contradiction. Then we could build a waitfree consensus algorithm for two processes $p_{0}$ and $p_{1}$ from that same collection of eventually linearizable objects and linearizable registers. Consider the tree of all possible histories of this consensus algorithm in which process $p_{i}$ has input $i$ for $i=0,1$. (In this tree, nodes represent configurations of the algorithm and the edge connecting a parent to a child are labelled by an invocation or response on one of the base objects.) A node $C$ is 0 -valent (or 1valent) if all decisions made in the subtree rooted at $C$ are 0 (or 1 , respectively). A node is multivalent if it is neither 0 - nor 1-valent. The root of this tree is multivalent, since a solo run by $p_{i}$ must produce output $i$. So there must be a critical configuration $C$ (i.e., $C$ is multivalent, but each child of $C$ in the tree is 0 - or 1-valent).

Thus, there must be one event $s_{0}$ at $p_{0}$ and one event $s_{1}$ at $p_{1}$ that take the system from $C$ to configurations $C_{0}$ and $C_{1}$ with opposite valencies. If $s_{0}$ and $s_{1}$ do not involve the same shared object $o$, then the events commute: the configurations reached from $C$ by doing $s_{0} s_{1}$ or $s_{1} s_{0}$ are indistinguishable to all processes. So, a solo execution by $p_{0}$ from either one leads to the same outcome, contradicting the fact that $C_{0}$ and $C_{1}$ have opposite valencies. We consider three cases.

CASE $1\left(s_{0}\right.$ is an invocation event on $o$ ): Let $\alpha$ be any solo run by $p_{1}$ continuing from $C_{1}$ until $p_{1}$ decides. Then $s_{1} \alpha$ is also a legal continuation from the configuration $C_{0}$, contradicting the fact that $C_{0}$ and $C_{1}$ have opposite valencies.

CASE $2\left(s_{1}\right.$ is an invocation event on $\left.o\right)$ : symmetric to Case 1.

CASE $3\left(s_{0}\right.$ and $s_{1}$ are both response events from $o$ ): We show $s_{0} s_{1}$ and $s_{1} s_{0}$ could both occur after $C$.

First, suppose $o$ is a linearizable register. Then, whatever response $p_{0}$ gets in event $s_{0}$ from configuration $C$ is still a valid response to $p_{0}$ 's pending operation after $s_{1}$ occurs. In particular, if $s_{0}$ returns a null response to a write by $p_{0}$, the write would receive exactly the same response after $s_{1}$. Likewise, if $s_{0}$ returns a value $v$ as the response to a read by $p_{0}$, that value is still a valid response to the read if $p_{1}$ gets the response to its operation first. So, $s_{1} s_{0}$ is a valid sequence of events following $C$. Similarly, $s_{0} s_{1}$ is a valid sequence of events following $C$.

Now suppose $o$ is an eventually linearizable object, then there is no constraint that the responses from $o$ in any finite prefix of the execution must satisfy (beyond weak consistency). So $s_{0} s_{1}$ and $s_{1} s_{0}$ are both possible sequences of events following $C$.
Let $s_{0} s_{1} \alpha$ be an execution onward from $C$ where $\alpha$ is a solo execution by $p_{0}$ until it decides. Then, $s_{1} s_{0} \alpha$ is a possible execution from $C$, contradicting the fact that $C_{0}$ and $C_{1}$ have opposite valencies.

\section{Implementing Eventually Linearizable Objects Using Registers}

We next consider whether eventually linearizable objects are easier to implement than their linearizable counterparts. Here, we focus on implementations from registers: we show that it is possible to obtain eventually linearizable implementations of some objects that have no linearizable implementations.

Any one-shot type has a trivial eventually linearizable implementation using no shared objects since the implementation may behave badly during the finite prefix when all operations are performed. Similarly, some long-lived types whose behaviour is "interesting" only in a finite prefix of each execution have eventually linearizable implementations using no shared memory. For example, a testEset object has an eventually linearizable implementation where each process simply returns 0 for its first invocation of test\&set and 1 for all subsequent invocations.

A consensus object provides one operation propose $(v)$, where $v$ is a value drawn from some domain $D$. Each propose operation returns the value used as the argument of the first propose operation to be linearized. This object is essentially the hardest object to implement in a linearizable way (since it can be used to build linearizable implementations of every other type [9]), but it is trivial to implement it in an eventually linearizable way using only linearizable registers. In fact, the following proposition states that such an implementation can be built even from eventually linearizable registers.

Proposition 16. A consensus object has a wait-free, eventually linearizable implementation from eventually linearizable registers.

Proof. The implementation for $n$ processes uses an array Proposals [1..n] of single-writer multi-reader registers, each initially holding the value $\perp \notin D$. When process $p_{i}$ invokes Propose $(v)$, it runs the following programme.

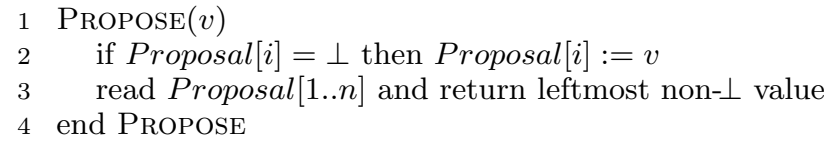

The weak consistency property of the base registers ensures that $p_{i}$ 's first read of Proposal [i] returns $\perp$ and all of $p_{i}$ 's subsequent reads of Proposal [i] returns the argument of $p_{i}$ 's first Propose operation. Thus, $p_{i}$ always sees a non- $\perp$ value on line 3 , so the leftmost non- $\perp$ value is well-defined. Moreover, if $p_{i}$ performs a write, then it only performs one write (during its first execution of Propose); all subsequent executions of line 2 must see a non- $\perp$ value in Proposal $i]$.

The implementation is clearly wait-free. Consider any concurrent execution of the implementation. Let $H$ be the history of invocations and responses on the consensus object during this execution and let $H^{\prime}$ be the history of invocations and responses on the base objects.

We first show that $H$ is weakly consistent. Let op be a Propose operation by $p_{i}$ that terminates in $H$ and returns the value $v$ it read from Proposal $[j]$. Then, the 
Propose $(v)$ operation $o p^{\prime}$ by process $p_{j}$ that wrote $v$ must have been invoked before op terminated. Thus, a sequential history that starts with $o p^{\prime}$ can be used to show that the result returned by op satisfies Definition 1 in $H$.

Our next goal is to define a value of $t$ such that $H$ is $t$-linearizable. By Lemma 7 , there is a $t^{\prime}$ such that $H^{\prime}$ is $t^{\prime}$ linearizable (since each of the finitely many base registers are eventually linearizable). Let $P$ be the set of PrOpose operations, all of whose reads terminate after event $t^{\prime}$ in $H^{\prime}$ and appear after all writes in the $t^{\prime}$-linearization of $H^{\prime}$. (Note that there are only finitely many Propose operations that are not in $P$ because there are at most $n$ writes during the execution.) Then, all Propose operations in $P$ read exactly the same set of values on line 3 and therefore return the same result $v_{0}$.

Choose $t$ such that a PROPOSE $\left(v_{0}\right)$ operation $o p_{0}$ begins before event $t$ of $H$ and the finitely many terminating Propose operations that are not in $P$ all terminate before event $t$ of $H$. To create a $t$-linearization of $H$, we put $o p_{0}$ first, followed by all other complete operations, in the order that they terminate. In this sequential history, all operations return $v_{0}$, as do all operations in the concurrent history that terminate after step $t$.

\section{EVENTUAL LINEARIZABILITY CAN BE HARD TO IMPLEMENT}

We prove in Proposition 18, below, that if we have an eventually linearizable implementation of a fetch\&increment object from linearizable base objects, we can "fix" the implementation so that it is linearizable. We use the following technical lemma.

Lemma 17. Suppose we have an eventually linearizable implementation of a fetchEincrement object. Let $t>0$ and let $\alpha$ be an infinite history of this implementation. If every finite prefix of $\alpha$ is t-linearizable, then $\alpha$ is $t$ linearizable.

Proof. Since the implementation is eventually linearizable, there is some $t^{\prime}$ such that $\alpha$ has a $t^{\prime}$-linearization $S^{\prime}$. If $t^{\prime} \leq t$, then the claim follows from Lemma 5. So, for the remainder of the proof, we assume $t^{\prime}>t$.

We partition the operations of $\alpha$ into four sets according to their response events as follows.

- Let $A_{1}$ contain the operations whose response is among the first $t$ events of $\alpha$.

- Let $A_{2}$ contain the operations whose response is among events $t+1, \ldots, t^{\prime}$.

- Let $A_{3}$ contain the operations whose response occurs after event $t^{\prime}$

- Let $A_{4}$ contain the operations that do not terminate.

Our goal is to construct a $t$-linearization $S$ of $\alpha$. We describe how to do this by assigning operations to positions in $S$. We assume the positions are numbered starting from 0 . If an operation in $A_{2}$ or $A_{3}$ returns a value $v$, we assign it to position $v$ in $S$. (Note that this cannot assign two operations to the same position, since if two operations $o p_{1}$ and $o p_{2}$ return the same result $v$, the prefix of $\alpha$ that includes the responses of both $o p_{1}$ and $o p_{2}$ would not be $t$-linearizable.) Let $E=\{v$ : no operation in $A_{2}$ or $A_{3}$ returns $\left.v\right\}$. This is the set of slots that have not yet been assigned an operation.

We first show that $\left|A_{1}\right| \leq|E| \leq\left|A_{1}\right|+\left|A_{4}\right|$. Let $E^{\prime}=$ $\left\{v\right.$ : no operation in $A_{3}$ returns $\left.v\right\}$. Then, $S^{\prime}$ fills all po- sitions of $E^{\prime}$ with operations in $A_{1}$ and $A_{2}$ and some subset $A_{4}^{\prime} \subseteq A_{4}$. Thus, $\left|A_{1}\right|+\left|A_{2}\right| \leq\left|E^{\prime}\right| \leq\left|A_{1}\right|+\left|A_{2}\right|+\left|A_{4}\right|$ (and all of these quantities are finite). Each operation of $A_{3}$ is assigned the same slot by $S$ and $S^{\prime}$ (since both are $t^{\prime}$-linearizations of $\alpha$ ). So, $|E|=\left|E^{\prime}\right|-\left|A_{2}\right|$. Thus, $\left|A_{1}\right| \leq|E| \leq\left|A_{1}\right|+\left|A_{4}\right|$.

For our linearization $S$, we fill in the first $\left|A_{1}\right|$ slots of $E$ with operations in $A_{1}$, in the order they are invoked in $\alpha$. Then, we fill in the remaining slots with the first $|E|-\left|A_{1}\right| \leq\left|A_{4}\right|$ operations of $A_{4}$ in the order of their invocations in $\alpha$. It remains to prove that $S$ is a $t$-linearization of $\alpha$.

- Each operation invoked in $S$ is invoked in $\alpha$ : This follows from the definition of $S$.

- Each operation that terminates in $\alpha$ appears in $S$ : By definition of $S$, all operations in $A_{1} \cup A_{2} \cup A_{3}$ are assigned slots in $S$.

- If $o p_{1}$ terminates after event $t$ and $o p_{2}$ begins after $o p_{1}$ terminates and $o p_{2}$ appears in $S$, then $o p_{1}$ precedes $o p_{2}$ in $S$ : Then, $o p_{1} \in A_{2} \cup A_{3}$ and $o p_{2} \in$ $A_{2} \cup A_{3} \cup A_{4}$. We consider several cases.

First, suppose $o p_{2}$ is in $A_{2} \cup A_{3}$. Then, $o p_{1}$ must return a smaller value than $o p_{2}$, since the prefix containing the responses of both $o p_{1}$ and $o p_{2}$ is $t$ linearizable. Thus, $o p_{1}$ is assigned an earlier slot than $o p_{2}$ in $S$.

Now, suppose $o p_{2}$ is in $A_{4}$. Let $v$ be the response returned by $o p_{1}$. Consider a prefix of $\alpha$ that contains all response events of $\alpha$ that return values less than or equal to $v$. By the hypothesis of the lemma, there is a $t$-linearization of this prefix. In that $t$-linearization, all the slots of $E$ prior to slot $v$ are filled using operations of $A_{1}$ and operations of $A_{4}$ that begin before $o p_{1}$ 's response (and hence before $o p_{2}$ is invoked). This means that the number of operations in $A_{1}$ and the number of operations in $A_{4}$ that begin before $o p_{2}$ begins is at least $|E \cap\{0,1, \ldots, v\}|$. In $S$, all of these operations are linearized before $o p_{2}$, so $o p_{2}$ is assigned a slot greater than $v$. Thus, $o p_{1}$ precedes $o p_{2}$ in $S$.

- Each operation that has a response after event $t$ of $\alpha$ has the same response in $S$ : Each operation of $A_{2} \cup A_{3}$ is assigned to the slot that would cause it to return the same response in $S$ as it does in $\alpha$.

For Lemma 17, the hypothesis that the implementation is eventually linearizable is necessary since $t$-linearizability is not a safety property of fetch\&increment implementations (see Section 3.2).

Proposition 18. If there is an n-process eventually linearizable, non-blocking implementation of a fetchछincrement object from a set $\mathcal{O}$ of linearizable objects, then there is an n-process linearizable, non-blocking implementation of a fetchछincrement object from $\mathcal{O}$.

Proof. Let $A$ be an $n$-process eventually linearizable implementation of a fetch\&increment object from $\mathcal{O}$. Consider the execution tree of all possible executions of this implementation in which $n$ processes $p_{1}, \ldots, p_{n}$ each repeatedly perform fetch\&inc operations forever. Each edge 
in the tree represents either a local event or an atomic action on an object in $\mathcal{O}$.

Consider a node $C$ in this tree. Let $\alpha_{C}$ be the finite execution represented by the path from the root to $C$. We say that $C$ is stable if every execution with prefix $\alpha_{C}$ is $\left|\alpha_{C}\right|$-linearizable. (In other words, the behaviour of the implemented fetch\&increment object has "stabilized" by $C$.)

It follows from the definition of stable and Lemma 5 that if $C$ is stable, then so are $C$ 's descendants.

Claim 1: There is a stable node in the tree.

Proof of Claim 1: To derive a contradiction, suppose no node in the tree is stable. We inductively construct a sequence of finite paths $p_{0}, p_{1}, \ldots$ that have the following properties, where $\ell_{i}=\left|p_{0} p_{1} \ldots p_{i}\right|$ :

1. For $i \geq 0, p_{0} p_{1} \cdots p_{i}$ is a path starting at the root of the tree.

2 . For $i \geq 1, p_{i}$ is non-empty.

3. For $i \geq 1, p_{0} p_{1} \ldots p_{i}$ is not $\ell_{i-1}$-linearizable.

Let $p_{0}$ be the empty execution. For the inductive step of the construction, let $i \geq 1$. Given $p_{0}, \ldots, p_{i-1}$ with the properties listed above, we construct $p_{i}$ as follows.

Let $C$ be the endpoint of the path $p_{0} p_{1} \ldots p_{i-1}$. By the assumption, $C$ is not stable. So, there is some execution $\alpha$ with prefix $p_{0} p_{1} \ldots p_{i-1}$ that is not $\ell_{i-1}$-linearizable. By Lemma 17, some finite prefix $\alpha^{\prime}$ of $\alpha$ is not $\ell_{i-1^{-}}$ linearizable. That prefix has length greater than $\ell_{i-1}$, so let $p_{i}$ be the non-empty path such that $\alpha^{\prime}=p_{0} p_{1} \ldots p_{i-1} p_{i}$.

Now, consider the infinite execution $\pi=p_{0} p_{1} \ldots$ Since the implementation is eventually linearizable, there is a $t$ such that this execution is $t$-linearizable. Choose $i$ such that $\ell_{i-1}>t$. (Such a choice is possible because all of the $p_{j}$ 's are non-empty, so we have $\ell_{0}<\ell_{1}<\cdots$.) Since $\pi$ is $t$-linearizable, it is also $\ell_{i-1}$ linearizable, by Lemma 5 . By Lemma 6 , the prefix $p_{0} p_{1} \ldots p_{i}$ of $\pi$ is also $\ell_{i-1}$ linearizable, which contradicts the third property of the construction. This completes the proof of Claim 1.

Let $C$ be a stable node. Let $t$ be the number of events in the execution $\alpha_{C}$. Consider the configuration $C_{i d l e}$ reached from $C$ by allowing each process to run solo until it completes its current fetch\&inc operation. Then, we let one process $p$ run fetch\&inc operations repeatedly. We argue that, eventually, some operation $o p_{0}$ that $p$ performs must return a value that is equal to the number of fetch\&inc operations that were invoked before $o p_{0}$. (If not, there would be no way to $t$-linearize the infinite execution where $p$ continues forever.) Let $C_{0}$ be the configuration at the end of $o p_{0}$. Let $v_{0}$ be the number of fetch\&inc operations invoked in the path from the root to $C_{0}$.

Now, we construct a linearizable implementation $A^{\prime}$ of a fetch\&increment object using $\mathcal{O}$. Initialize each object in $\mathcal{O}$ to the state it has in $C_{0}$. Similarly, each process uses the same local variables as in $A$, and they are initialized in $A^{\prime}$ to the values they have in $C_{0}$. In $A^{\prime}$, to perform a fetch\&inc operation, a process executes the algorithm $A$ for fetch\&inc until it obtains a result $v$, and then it returns $v-v_{0}$.

If $\beta$ is an execution of $A^{\prime}$, there is a corresponding execution $\alpha \beta^{\prime}$ of $A$, where $\alpha$ is the execution described above that takes the system to configuration $C_{0}$, and $\beta^{\prime}$ is the same as $\beta$ except that $v_{0}$ is not subtracted from the output values. Since $C$ is stable, $\alpha \beta^{\prime}$ is $t$-linearizable, and the $t$-linearization must linearize before $o p_{0}$ all $v_{0}$ operations invoked before $o p_{0}$. Moreover, the $t$-linearization respects the real-time order of operations in $\beta^{\prime}$ since they are invoked after event $t$ of $\alpha \beta^{\prime}$. The suffix of the $t$-linearization obtained by removing the first $v_{0}$ operations is a linearization of all operations in $\beta$.

Remark: The preceding proposition can also be proved for other progress conditions: if the eventually linearizable implementation is obstruction-free or wait-free, then it can be used to create an obstruction-free or wait-free linearizable implementation.

COROllary 19. There is no non-blocking eventually linearizable implementation of a fetchEincrement object for two processes from linearizable registers.

Proof. If there were such an implementation, we could build a linearizable implementation of a fetch\&increment object for two processes from linearizable registers, by Proposition 18. This is impossible since a fetch\&increment object and registers can solve two-process consensus and registers alone cannot $[9,12]$.

\section{OPEN QUESTIONS}

We have shown that eventually linearizable implementations can be much easier to build (and weaker) than linearizable ones for some types (e.g., consensus objects). For other types (such as fetch\&increment), an eventually linearizable implementation appears to be just as hard to build as a linearizable one. It would be interesting to characterize the exact situations where an eventually linearizable implementation is easier to attain than a linearizable one.

One of the fundamental results about linearizable objects is Herlihy's wait-free universal construction [9]. It is natural to ask whether there is a lock-free universal construction of eventually linearizable objects from some natural eventually linearizable primitive objects (possibly in conjunction with linearizable registers). The results of [5] may provide some ideas for doing this.

\section{APPENDIX: PROOF OF PROPOSITION 11}

Proof. The "only if" direction is trivial by the definition of eventually linearizable. It remains to prove the "if" direction. Consider a system of $n$ processes $p_{1}, \ldots, p_{n}$. Let $A$ be an implementation of $T$ with the property that for every history there is a $t$ such that the history is $t$ linearizable. We define a new implementation $A^{\prime}$ that is eventually linearizable.

In addition to whatever shared objects are used by implementation $A, A^{\prime}$ uses a sequence of single-writer registers $R_{i}[0,1,2, \ldots]$ for each process $p_{i}$. All of these additional registers are initialized to the value $\perp$. Each process $p_{i}$ also stores a local counter $c_{i}$, initially 0 , that counts how many operations $p_{i}$ has performed, and a local variable $q_{i}$ (initialized to the initial state of the implemented object) that stores the state of the implemented object that would result if only $p_{i}$ 's own operations were performed on it. In the implementation $A^{\prime}, p_{i}$ performs an operation op by executing the algorithm in Figure 1 .

First, we prove $A^{\prime}$ is non-blocking. The call to algorithm $A$ on line 5 is non-blocking, by the hypothesis. The 


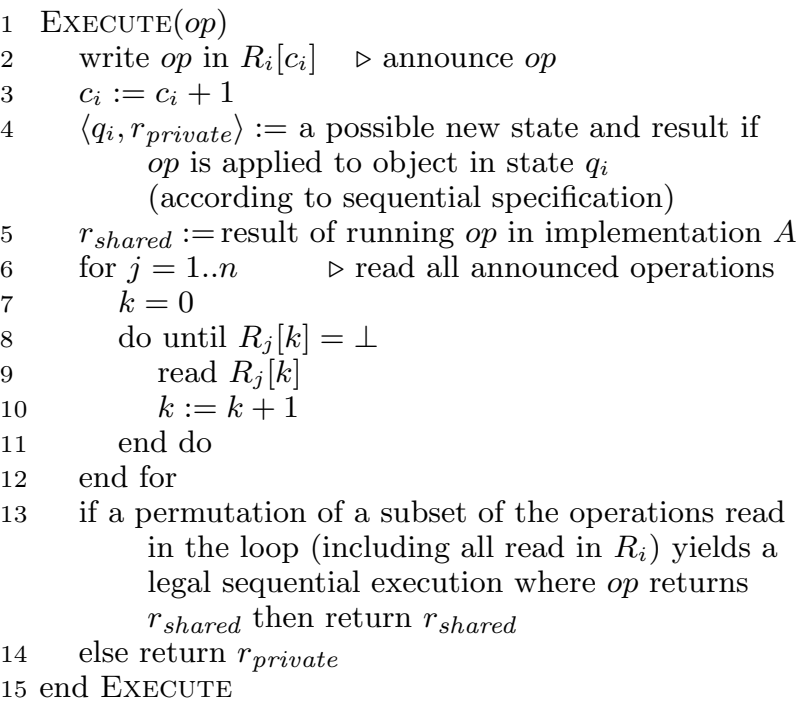

Figure 1: Algorithm to guarantee weak consistency.

only way the loop (line 8-11) can run forever without terminating is if process $p_{j}$ increments its counter $c_{j}$ infinitely many times, meaning that $p_{j}$ completes infinitely many operations. Also, there are only finitely many permutations to try in line 13 , since $T$ has finite nondeterminism.

We now show that any history $H$ generated by this implementation is eventually linearizable.

Consider an operation op that terminates in $H$ and is performed by some process $p$. If op returns on line 14, let $S_{\text {private }}$ be the sequential execution defined by the sequence of operations performed by $p$ up to and including $o p$, with the responses chosen on line 4 . Then $S_{\text {private }}$ satisfies Definition 1 for $o p$ in $H$. If $o p$ returns on line 13 , then the permutation described on line 13 defines a sequential execution $S_{\text {shared }}$ that satisfies Definition 1 for $o p$ in $H$. (Note that all operations included in the permutation begin before $o p$ terminates.) Thus, $H$ is weakly consistent.

Next, we prove that $H$ is $t$-linearizable for some $t$. Consider the execution that consists of all steps inside the calls to implementation $A$ on line 5 . These define a history $H_{A}$. By the hypothesis about implementation $A, H_{A}$ is $t_{A}$-linearizable for some $t_{A}$. Let $S$ be a $t_{A}$-linearization of $H_{A}$.

Let $O$ be the set of operations $o p$ such that $o p$ terminates in $H$ and $o p$ 's call to $A$ on line 5 terminates within the first $t_{A}$ events in $H_{A}$. Note that $O$ is finite, because only finitely many operations terminate before event $t_{A}$ in $H_{A}$. Choose $t$ large enough that that all operations in $O$ terminate before event $t$ of $H$. (This is possible because $O$ is a finite set of operations, all of which terminate in $H$.) We prove that the legal sequential execution $S$ is a $t$-linearization of $H$ by showing it satisfies the four properties in Definition 2.

Since $S$ is a $t_{A}$-linearization of $H_{A}$, each operation invoked in $S$ is also invoked in $H_{A}$, and is therefore invoked in $H$.
Each operation that terminates in $H$ also must terminate in $H_{A}$, and therefore terminates in $S$ (since $S$ is a $t_{A}$-linearization of $H_{A}$ ).

Consider any two operations $o p_{1}$ and $o p_{2}$ such that $o p_{1}$ terminates before $o p_{2}$ is invoked in $H$ and both of these events occur after event $t$ of $H$, and $o p_{2}$ is in $S$. Let $o p_{1}^{\prime}$ and $o p_{2}^{\prime}$ be the operations in $H_{A}$ called at line 5 of $o p_{1}$ and $o p_{2}$, respectively. Note that $o p_{1}^{\prime}$ exists because $o p_{1}$ terminates, and $o p_{2}^{\prime}$ exists because the operation appears in $S$, which is a $t_{A}$-linearization of $H_{A}$. Since $o p_{1}$ terminates after event $t$ of $H, o p_{1}$ does not belong to $O$, so $o p_{1}^{\prime}$ terminates after the first $t_{A}$ events of $H_{A}$. Moreover, $o p_{2}$ 's call to $A$ on line 5 is after $o p_{2}$ begins, which is after $o p_{1}$ terminates, which is after $o p_{1}$ 's call to $A$ terminates. Thus, $o p_{1}^{\prime}$ terminates before $o p_{2}^{\prime}$ begins in $H_{A}$. Since $S$ is a $t_{A}$-linearization of $H_{A}, o p_{1}$ precedes $o p_{2}$ in $S$.

Suppose $o p$ is an operation that terminates in $H$ after the first $t$ events. We first argue that the test performed by $o p$ at line 13 evaluates to true, so that $o p$ returns $r_{\text {shared }}$ at line 13. Let $o p_{p}$ be any operation that precedes $o p$ in $S$. Let $o p^{\prime}$ and $o p_{p}^{\prime}$ be the operations in $H_{A}$ called at line 5 of $o p$ and $o p_{p}$, respectively. Note that $o p^{\prime}$ exists because $o p$ terminates, and $o p_{p}^{\prime}$ exists because it appears in $S$, which is a $t_{A}$-linearization of $H_{A}$. Since op terminates after event $t$ of $H$, op does not belong to $O$, so $o p^{\prime}$ terminates after the first $t_{A}$ events of $H_{A}$. So, $o p_{p}^{\prime}$ must begin before $o p^{\prime}$ terminates in $H_{A}$ (otherwise, $o p$ would have to precede $o p_{p}$ in $S$ ). This means that $o p_{p}$ begins executing line 5 before op finishes executing line 5 . So, $o p_{p}$ executes line 2 before $o p$ reaches line 6 . Since this is true for every operation $o p_{p}$ that precedes $o p$ in $S, o p$ will see the announcement of every operation that precedes $o p$ in $S$ when it reads the registers. Thus, one possible permutation that $o p$ considers in the test on line 13 will be the prefix of $S$ up to $o p$, which yields the response $r_{\text {shared }}$ for $o p$, so the test will evaluate to true, and $o p$ will return $r_{\text {shared }}$, which is the same value that op returns in $S$.

\section{REFERENCES}

[1] P. Bailis and A. Ghodsi. Eventual consistency today: Limitations, extensions and beyond. Commun. ACM, 56(5):55-63, May 2013.

[2] A. Bouajjani, C. Enea, and J. Hamza. Verifying eventual consistency of optimistic replication systems. In Proc. 41st ACM Symposium on Principles of Programming Languages, pages 285-296, 2014.

[3] C. Delporte-Gallet, H. Fauconnier, and R. Guerraoui. Tight failure detection bounds on atomic object implementations. J. ACM, 57(4), Apr. 2010.

[4] A. Demers, D. Greene, C. Hauser, W. Irish, J. Larson, S. Shenker, H. Sturgis, D. Swinehart, and D. Terry. Epidemic algorithms for replicated database maintenance. In Proc. 6th ACM Symposium on Principles of Distributed Computing, pages 1-12, 1987.

[5] S. Dubois, R. Guerraoui, P. Kouznetsov, F. Petit, and P. Sens. The weakest failure detector for eventual consistency. Unpublished manuscript, 2014.

[6] A. Fekete, D. Gupta, V. Luchangco, N. Lynch, and A. Shvartsman. Eventually-serializable data services. Theoretical Comput. Sci., 220(1):113-156, June 1999. 
[7] M. J. Fischer, N. A. Lynch, and M. S. Paterson. Impossibility of distributed consensus with one faulty process. J. ACM, 32(2):374-382, Apr. 1985.

[8] R. Guerraoui and E. Ruppert. Linearizability is not always a safety property. In Proc. 2nd International Conference on Networked Systems, 2014. To appear.

[9] M. Herlihy. Wait-free synchronization. ACM Trans. Prog. Lang. Syst., 13(1):124-149, Jan. 1991.

[10] M. Herlihy and N. Shavit. On the nature of progress. In Proc. 15th International Conference on Principles of Distributed Systems, pages 313-328, 2011.

[11] M. P. Herlihy and J. M. Wing. Linearizability: a correctness condition for concurrent objects. ACM Trans. Prog. Lang. Syst., 12(3):463-492, July 1990.

[12] M. C. Loui and H. H. Abu-Amara. Memory requirements for agreement among unreliable asynchronous processes. In F. P. Preparata, editor, Advances in Computing Research, volume 4, pages 163-183. JAI Press, Greenwich, Connecticut, 1987.

[13] N. Lynch. Distributed Algorithms, chapter 13. Morgan Kaufmann, 1996.

[14] Y. Saito and M. Shapiro. Optimistic replication.
ACM Computing Surveys, 37(1):42-81, Mar. 2005.

[15] A. G. Sebastien Burckhard and H. Yang. Understanding eventual consistency. Technical report, Microsoft, 2013.

[16] M. Serafini, D. Dobre, M. Majuntke, P. Bokor, and N. Suri. Eventually linearizable shared objects. In Proc. 29th ACM Symposium on Principles of Distributed Computing, pages 95-104, 2010. Full version available from http://labs.yahoo.com/files/aurora.pdf.

[17] A. Singh, P. Fonseca, P. Kuznetsov, R. Rodrigues, and P. Maniatis. Zeno: Eventually consistent byzantine-fault tolerance. In Proc. 6th USENIX Symposium on Networked Systems Design and Implementation, pages 169-184, 2009.

[18] D. B. Terry, M. M. Theimer, K. Petersen, A. J. Demers, M. J. Spreitzer, and C. H. Hauser. Managing update conflicts in Bayou, a weakly connected replicated storage system. In Proc. 15th ACM Symposium on Operating Systems Principles, pages 172-182, 1995.

[19] W. Vogels. Eventually consistent. Commun. ACM, 52(1):40-44, Jan. 2009. 